\title{
Managed Care im Kanton Thurgau - eine Erfolgsgeschichte
}

\author{
Seit über 10 Jahren engagieren sich die Thurgauer Ärztinnen und Ärzte für \\ Managed Care und haben die gesamtschweizerische Entwicklung mass- \\ gebend mitgeprägt. Das Erfolgsmodell hat die Ärzteschaft in verschiedenen \\ anderen Regionen dazu motiviert, eigene Ärztenetzwerke zu gründen, und \\ dazu beigetragen, die politische Diskussion zu öffnen. Die Thurgauer Ärzte- \\ netzwerke sind heute fester Bestandteil in der regionalen Gesundheitsversor- \\ gung. Bereits $30 \%$ der Bevölkerung sind in einem verbindlichen Managed- \\ Care-Modell versichert.
}

R. Buff, A. Schneider,

P. Sauter, K. Metzger

1 Buff R, Kappeler O, Schneider A Niemann M, Metzger K. ManagedCare-Entwicklung im Kanton Thurgau. Schweiz Ärztezeitung. 2002;83(51/52):2830-5.

Korrespondenz:

Verband Managed Care Thurgau

c/o thurcare AG

Zürcherstrasse 112

CH-8500 Frauenfeld

\section{Meilensteine der Entwicklung [1]}

Seit 1995 ist die Thurgauer Ärzteschaft gewillt, das regional geprägte Gesundheitssystem aktiv mitzugestalten. 1996 haben die ersten Thurgauer Hausarztvereine mit ersten Versicherern Hausarztmodellverträge abgeschlossen.

1997 wurde unter dem Titel «Auf dem Weg vom Hausarztmodell zum Managed-CareSystem» unter der Leitung der Thurgauer Ärztegesellschaft und mit Einbezug aller ärztlichen Interessengruppen (Hausärzte, Spezialisten, Spitalärzte) die Voraussetzungen für «integrierte Versorgungsnetze» geschaffen. In einem Grundlagenpapier sind die Eckwerte für ManagedCare-Systeme, Verhaltensregeln zur Zusammenarbeit zwischen Hausärzten, Spezialisten und Spitalärzten erarbeitet und verabschiedet worden. Darauf aufbauend erfolgte die Entwicklung vom Hausarztmodell hin zum Thurgauer ManagedCare-System zielgerichtet und einvernehmlich.

Mit der Gründung eines eigenen ärztlichen Dienstleistungs- und Kompetenzzentrums - der thurcare AG - haben sich die vier Thurgauer Ärztenetzwerke 1998 die notwendigen professionellen Strukturen gegeben, um der Komplexität und den steigenden Ansprüchen gerecht zu werden sowie auch nichtärztliche Kompetenzen zu entwickeln.

Als überregionale Dachorganisation der Ärztenetzwerke wurde im 2001 der Verband Managed Care Thurgau (vmct) gegründet. Dieser koordiniert und vertritt die Ärztenetzwerke nach aussen.

\section{Status quo}

Im Thurgau sind heute aus den ursprünglich sieben regionalen Hausarztvereinen vier integrierte Ärztenetzwerke mit knapp 190 engagierten Ärzten (Grundversorger, Spezialisten, Spitalärzte) und 66000 Managed-Care-Versicherten entstanden.

thurcare hat sich als führender Anbieter von Dienstleistungen im Bereich Managed Care in der Ostschweiz etabliert und unterstützt auch Ärztenetzwerke im Kanton St. Gallen: Rhymed, St. Galler Rheintal; HAV St. Gallen, Stadt St. Gallen und xundart AG, Region Wil-Uzwil-Toggenburg. thurcare stellt die administrative Abwicklung mit den Ärzten und Versicherern und das Datenmanagement mit dem Informationssystem BlueEvidence sicher.

\section{Ausblick}

In den Netzwerkleitungen ist eine neue, junge Generation von Medizinern tätig, die die Ideen und Visionen der Thurgauer Managed-CarePioniere konsequent weiterträgt. Themen wie die Qualitätssteigerung oder die Erhöhung der Netzwerkverbindlichkeit werden fortwährend bearbeitet und stehen stellvertretend für diese erfreuliche Entwicklung. Im vergangenen Jahr haben die Delegierten des Dachverbands der Thurgauer Ärztenetzwerke (vmct) beschlossen, die Strukturen zu öffnen und die Weiterentwicklung kantonsüberschreitend zu fördern. 2008 entsteht aus dem Verband Managed Care Thurgau (vmct) der Verband Managed Care Ostschweiz (vmco).

Damit soll das nächste Kapitel der Thurgauer hin zu einer Ostschweizer Erfolgsgeschichte geschrieben werden. 